\title{
Diffusion-thermo and Aligned Magnetic Field Effects on Free Convection on Flow past an Inclined Porous Plate with First Order Chemical Reaction
}

\author{
${ }^{1}$ G. Sreenivasulu Reddy, ${ }^{2}$ S. Karunakar Reddy, ${ }^{* 3}$ P.Durga Prasad and \\ ${ }^{4}$ S.Vijayakumar Varma \\ ${ }^{1}$ Reader in Mathematics, Govt. Degree College, Pakala, Chittoor, A.P, India \\ ${ }^{2}$ Department of Mathematics, JNTU Hyderabad, Telangana \\ ${ }^{3,4}$ Department of Mathematics, S.V.University, Tirupati
}

\begin{abstract}
A theoretical analysis is carried out to study the heat and mass transfer flow of viscous incompressible fluid past an inclined porous plate with the aligned magnetic field and Diffusion-thermo effects. A uniform magnetic field of strength $B_{0}$ is applied at an angle $\psi$ with the fluid flow direction. The analytical solutions to the coupled non-linear equations governing the motion are obtained by regular perturbation technique. The effects various material parameters on velocity, temperature, concentration, the rate of mass transfer, the rate of heat transfer and the skin-friction coefficients have been analyzed through graphs and table.

Keywords: Diffusion-thermo effect; Magnetic field; Thermal Radiation; Chemical reaction; Free convection.
\end{abstract}

\section{Introduction}

The study of convective heat transfer from a solid body with different geometries embedded in a fluid saturated porous medium has varied and wide applications in many areas of science and engineering such as geothermal reservoirs, drying of porous solids, chemical catalytic reactors, thermal insulators, nuclear waste repositories, heat exchanger devices, enhanced oil and gas recovery, and underground energy transport. An investigation of an influence of magnetic field on viscous incompressible flow of electrically conducting fluid has its importance in many applications such as extrusion of plastics in the manufacture of rayon and nylon, paper industry, and textile industry and in different geophysical cases and so forth. A comprehensive review of the studies of convective heat transfer mechanism through porous medium has been made by Nield and Bejan [10]. Conjugate effects of heat and mass transfer on MHD free convection flow over an inclined plate embedded in a porous medium was studied by Farhad [2]. Convection effects on flow past an inclined plate with variable surface temperatures in water at $4{ }^{\circ} \mathrm{C}$ has been studied by Palani [3]. Barik et al. [4] have studied the thermal radiation effects on an unsteady MHD flow past an inclined porous heated plate in the presence of chemical reaction and viscous dissipation. Chen [5] studied heat and mass transfer in MHD flow by natural convection from a permeable inclined surface with variable wall temperature and concentration. Reddy et al. [6] have discussed the effects of thermal radiation on an unsteady magnetohydrodynamic flow past an inclined porous heated plate in the presence of chemical reaction with heat and mass transfer by using a closed analytical form method. Ganesan and Palani [7] have studied the problem of unsteady natural convection flow of a viscous incompressible electrically conducting fluid past an inclined plate with variable heat and mass flux's. Reddy [11] studied the mass transfer and heat generation effects on MHD free convection flow past an inclined vertical surface in a porous medium. Very recently, Prasad [17] studied diffusion-thermo and porous medium effects on MHD free convection heat and mass transfer flow over an inclined surface.

Gribben [1] investigated the MHD boundary layer flow over a semi-infinite plate with an aligned magnetic field in the presence of pressure gradient. Convective heat and mass transfer flow of chemically reactive and radiation absorption fluid in an aligned magnetic field was studied by Prabhakara Reddy [8]. Unsteady flow and heat transfer on a semi-infinite flat plate with an aligned magnetic field was studied by Takhar [9].

The interaction of buoyancy with thermal radiation has in increased greatly during the last decade due to its importance in many practical applications. The thermal radiation effect is important under many isothermal and non-isothermal situations. If the entire system involving the polymer extrusion process is placed in a thermally controlled environment, then thermal radiation could be important. The knowledge of radiation heat transfer in the system can perhaps, lead to a desired product with sought characteristics.

In most of the studies related to heat and mass transfer process, Soret and Dufour effects are neglected on the basis that they are of a smaller order of magnitude than the effects described by Fouriers and Ficks laws. But these effects are considered as second order phenomena and may become significant in areas such as 
hydrology, petrology, geosciences, etc. The Dufour effect was found to be of order of considerable magnitude so that it cannot be neglected Eckert and Drake [12]. Due to the importance of Soret (thermal-diffusion) and Dufour (diffusion thermo) effects for the fluids with very light molecular weight as well as medium molecular weight many investigators have studied and reported results for these flows of whom the names are Dursunkaya and Worek [14], Anghel et.al. [13], are worth mentioning.

The above mentioned papers the diffusion thermo effect past an inclined surfaces with aligned magnetic field effects was not studied. So the objective of the present study is to investigate the effects of diffusion-thermo, chemical reaction effects, radiation absorption and heat absorbing fluid past a vertical permeable plate with aligned magnetic field bounded by an inclined surface subjected to the suction velocity varying with time.

\section{Mathematical Formulation}

In this work we considered an unsteady two-dimensional MHD flow of viscous incompressible and electrically conduction fluid past an inclined porous plate with an angle $\alpha$ and subjected to the aligned magnetic field strength $B_{0}$ in the presence of thermal and concentration buoyancy effects. $x$-axis is taken vertically upward direction along the plate and $y$-axis is normal to it. The magnetic Reynolds number is assumed to be small. It is assumed that there is no applied voltage which implies the absence of an electrical field. Also assumed that the Hall effects, electrical effects and polarization effects are neglected and the Joules dissipation term in the energy equation is neglected. Due to the semi-infinite plate surface assumption the flow variables are functions of $y^{*}$ and $t^{*}$ only. By using Boussinesq's approximation, the governing equations of the flow are given by

$\frac{\partial v^{*}}{\partial y^{*}}=0$

$\frac{\partial u^{*}}{\partial t^{*}}+v^{*} \frac{\partial u^{*}}{\partial y^{*}}=-\frac{1}{\rho} \frac{\partial p^{*}}{\partial x^{*}}+v \frac{\partial^{2} u^{*}}{\partial y^{*^{2}}}-\frac{\sigma B_{0}^{2} \sin ^{2} \psi}{\rho} u^{*}+g \beta_{T} \cos \alpha\left(T^{*}-T_{\infty}\right)+g \beta_{c} \cos \alpha$

$\frac{\partial T^{*}}{\partial t^{*}}+v^{*} \frac{\partial T^{*}}{\partial y^{*}}=\frac{K}{\rho C_{p}} \frac{\partial^{2} T^{*}}{\partial y^{* 2}}-\frac{1}{\rho C_{p}} \frac{\partial q_{r}^{*}}{\partial y^{*}}-\frac{Q_{0}}{\rho C_{p}}\left(T^{*}-T_{\infty}\right)+\frac{Q_{1}^{*}}{\rho C_{p}}\left(C^{*}-C_{\infty}\right)+\frac{D_{m} K_{T}}{C_{s} \rho C_{p}} \frac{\partial^{2} C^{*}}{\partial y^{*}}$

$\frac{\partial C^{*}}{\partial t^{*}}+v^{*} \frac{\partial C^{*}}{\partial y^{*}}=D \frac{\partial^{2} C^{*}}{\partial y^{*}}-R\left(C^{*}-C_{\infty}\right)$

Where $u^{*}$ and $v^{*}$ are the components of dimensional velocities along $x^{*}$ and $y^{*}$ directions, respectively, $T^{*}$ is the dimensional temperature, $C^{*}$ is the dimensional concentration, $T_{w}$ and $C_{w}$ are the temperature and concentration at the wall, $C_{\infty}$ and $T_{\infty}$ are the free stream dimensional concentration and temperature. $\rho$ is the density, $v$ is kinematic viscosity, $C_{p}$ is the specific heat at constant pressure, $\sigma$ is the fluid electrical conductivity, $B_{0}$ is the magnetic induction, $K^{*}$ is the permeability of the porous medium, $q_{r}^{*}$ is radiative heat flux, $Q_{0}$ is the dimensional heat absorption coefficient, $Q_{1}^{*}$ is the coefficient of proportionality for the absorption, $\mathrm{R}$ is the chemical reaction, $\beta_{T}$ and $\beta_{c}$ are the thermal and concentration expansion coefficients, $\mathrm{D}$ is the molecular diffusivity, $D_{m}$ is the coefficient of mass diffusivity, $K_{T}$ is the thermal diffusion ratio,

$Q_{0}\left(T^{*}-T_{\infty}\right)$ is assumed to be amount of heat generated or absorbed per unit volume $Q_{0}$ is a constant and $\alpha$ is an angle of inclination and $\Psi$ is aligned magnetic field angle.

The radiative heat flux is given by

$\frac{\partial q_{r}^{*}}{\partial y^{*}}=4\left(T^{*}-T_{\infty}\right) I^{\prime}$

where $I^{\prime}=\int_{0}^{\infty} K_{\lambda w} \frac{\partial e_{b \lambda}}{\partial T^{*}} d \lambda, K_{\lambda w}$ is the absorption coefficient at the wall and $e_{b \lambda}$ is Planck's function.

The boundary conditions for velocity involving slip flow, temperature and concentration fields are defined as

$u^{*}=u_{\text {slip }}^{*}=\frac{\sqrt{k}}{\alpha} \frac{\partial u^{*}}{\partial y^{*}}, T^{*}=T_{w}+\epsilon\left(T_{w}-T_{\infty}\right) e^{n^{*} t^{*}}$, at $y^{*}=0$

$C^{*}=C_{w}+\epsilon\left(C_{w}-C_{\infty}\right) e^{n^{*} t^{*}}$ at $y^{*}=0$

$u^{*}=U_{\infty}^{*}=U_{0}\left(1+\epsilon e^{n^{*} t^{*}}\right), T^{*} \rightarrow T_{\infty}, C^{*} \rightarrow C_{\infty}$, as $y^{*} \rightarrow \infty$

It is clear from (1) that the suction velocity at the plate surface is a function of time only. Assuming that, the suction velocity takes the following exponential form:

$\vartheta^{*}=-V_{0}\left(1+\epsilon A e^{n^{*} t^{*}}\right)$ 
$-\frac{1}{\rho} \frac{d p^{*}}{d x^{*}}=\frac{d U_{\infty}^{*}}{d t^{*}}+\frac{\sigma}{\rho} B_{0}^{2} U_{\infty}^{*}$

We know introduce the following dimensionless variables

$u=\frac{u^{*}}{U_{0}}, \vartheta=\frac{\vartheta^{*}}{V_{0}}, y=\frac{v_{0} y^{*}}{v}, U_{\infty}=\frac{U_{\infty}^{*}}{U_{0}}$,

$n=\frac{n^{*} v}{V_{0}^{2}}, G r=\frac{\rho g v\left(T_{w}-T_{\infty}\right) \beta_{T}}{U_{0} V_{0}^{2}}, \emptyset=\frac{Q_{0} v}{\rho V_{0}^{2} C_{p}}$,

$G m=\frac{\rho g v\left(C_{w}-C_{\infty}\right) \beta_{C}}{U_{0} V_{0}^{2}}, M=\frac{\sigma v B_{0}^{2}}{\rho V_{0}^{2}}, \quad \operatorname{Pr}=\frac{v C_{p}}{K}$,

$Q_{1}=\frac{Q_{1}^{*} v\left(C_{w}-C_{\infty}\right)}{V_{0}^{2}\left(T_{w}-T_{\infty}\right)}, F=\frac{4 v I^{\prime}}{V_{0}^{2} \rho C_{p}}, S c=\frac{v}{D}, D u=\frac{D_{m} k_{T}}{C_{S} K} \frac{\left(C_{w}-C_{\infty}\right)}{\left(T_{w}-T_{\infty}\right)}, \gamma=\frac{R v}{V_{0}^{2}}$

In the view of the above non-dimensional variables, the basic field of equations (2) to (4) can be expressed in non-dimensional form as

$$
\begin{aligned}
& \frac{\partial u}{\partial t}-\left(1+\epsilon A e^{n t}\right) \frac{\partial u}{\partial y}=\frac{d U_{\infty}}{d t}+M\left(U_{\infty}-U\right)+\frac{\partial^{2} u}{\partial y^{2}}+G r_{1} \theta+G m_{1} C, \\
& \frac{\partial \theta}{\partial t}-\left(1+\epsilon A e^{n t}\right) \frac{\partial \theta}{\partial y}=\frac{1}{P r} \frac{\partial^{2} \theta}{\partial y^{2}}-F \theta+Q_{1} C-\emptyset \theta+\frac{D_{u}}{P r}\left(\frac{\partial^{2} C}{\partial y^{2}}\right) \\
& \frac{\partial C}{\partial t}-\left(1+\epsilon A e^{n t}\right) \frac{\partial C}{\partial y}=\frac{1}{S c} \frac{\partial^{2} C}{\partial y^{2}}-\gamma C
\end{aligned}
$$

The corresponding boundary conditions (6)-(8) in dimensionless form are

$u=u_{\text {slip }}=\emptyset_{1} \frac{\partial u}{\partial y}, \theta=1+\epsilon e^{n t}, C=1+\epsilon e^{n t}$ at $\mathrm{y}=0$

$u \rightarrow U_{\infty}=\left(1+\epsilon e^{n t}\right), \theta \rightarrow 0, C \rightarrow 0$ as $y \rightarrow \infty$

Where $\emptyset_{1}=\frac{\sqrt{k}}{\alpha} \frac{U_{0} V_{0}}{v}$ is the slip parameter.

\section{Method Of Solution}

Equations (12) to (14) represent a set of partial differential equations that cannot be solved in closed form. However it can reduce to a set of ordinary differential in dimension less form that can be solved analytically. This can be done by representing the velocity, temperature and concentration as

$u=f_{0}(y)+\epsilon e^{n t} f_{1}(y)+O\left(\epsilon^{2}\right)$

$\theta=g_{0}(y)+\epsilon e^{n t} g_{1}(y)+O\left(\epsilon^{2}\right)$

$C=h_{0}(y)+\in e^{n t} h_{1}(y)+O\left(\epsilon^{2}\right)$

Substituting (17)-(19) into (12)-(14) and equating the harmonic and non-harmonic terms, and neglecting the higher order $O\left(\epsilon^{2}\right)$, and simplifying using the corresponding boundary conditions, we get

$u(y, t)=1+B_{4} e^{-m_{6} y}-A_{3} e^{-m_{2} y}-A_{2} e^{-P_{1} y}+\in e^{n t}\left(1+B_{5} e^{-m_{3} y}+A_{4} e^{-m_{6} y}-A_{10} e^{-m_{2} y}-B_{1} e^{-P_{1} y}-\right.$

$\left.B_{2} e^{-m_{5} y}-B_{3} e^{-m_{4} y}\right)$

$\theta(y, t)=\left(1-A_{1}\right) e^{-m_{2} y}+A_{1} e^{-P_{1} y}++\in e^{n t}\left(A_{9} e^{-m_{5} y}+A_{6} e^{-P_{1} y}+A_{7} e^{-m_{2} y}+A_{8} e^{-m_{4} y}\right)$

$C(y, t)=e^{-P_{1} y}++\in e^{n t}\left(\left(1-A_{5}\right) e^{-m_{4} y}+A_{5} e^{-P_{1} y}\right)$

The Skin-friction coefficient $C f$, the rate of heat transfer coefficient and the rate of mass transfer and are important physical parameters for this type of boundary layer flow which are defined and determined as follows:

$C_{f x}=\frac{\tau_{w}}{\rho U_{0} V_{0}}=\left(\frac{\partial \mathrm{u}}{\partial \mathrm{y}}\right)_{\text {at } y=0}$

$C_{f x}=-B_{4} m_{6}+A_{3} m_{2}+A_{2} P_{1}+\in e^{n t}\left(-B_{5} m_{3}-A_{4} m_{6}+A_{10} m_{2}+B_{1} P_{1}+B_{2} m_{5}+B_{3} m_{4}\right)$.

$N u_{x}=x \frac{\left(\frac{\partial T}{\partial y^{*}}\right)_{a t ~} y=0}{\left(T_{w}-T_{\infty}\right)} \Rightarrow N u_{x} / R e_{x}=\left(\frac{\partial \theta}{\partial y}\right)_{\text {at } y=0}$

$N u_{x} /_{R e_{x}}=\left(-m_{2}\left(1-A_{1}\right)-A_{1} P_{1}\right)+\in e^{n t}\left(-m_{5} A_{9}-P_{1} A_{6}-m_{2} A_{7}-m_{4} A_{8}\right)$ 
$S h_{x}=x \frac{\left(\frac{\partial C}{\partial y^{*}}\right)_{a t y=0}}{\left(C_{w}-C_{\infty}\right)} \Rightarrow S h_{x} / R e_{x}=\left(\frac{\partial C}{\partial y}\right)_{a t y=0}=$

$-P_{1}+\in e^{n t}\left(-m_{4}\left(1-A_{5}\right)-A_{5} P_{1}\right)$

Where $R e_{x}=\frac{V_{0} x}{v}$ is the local Reynolds number.

\section{Results and Discussion}

The solutions are performed for velocity, temperature, and concentration for various values of material parameters, the Skin friction coefficient, rate of heat transfer coefficient and the rate of mass transfer coefficient are presented from figures 1-15. Throughout the calculations the parametric values are chosen as $\mathrm{Pr}=$ $0.71, A=0.5, n=0.1, t=1, S c=0.6, \in=0.02, G r=4, G m=2$.

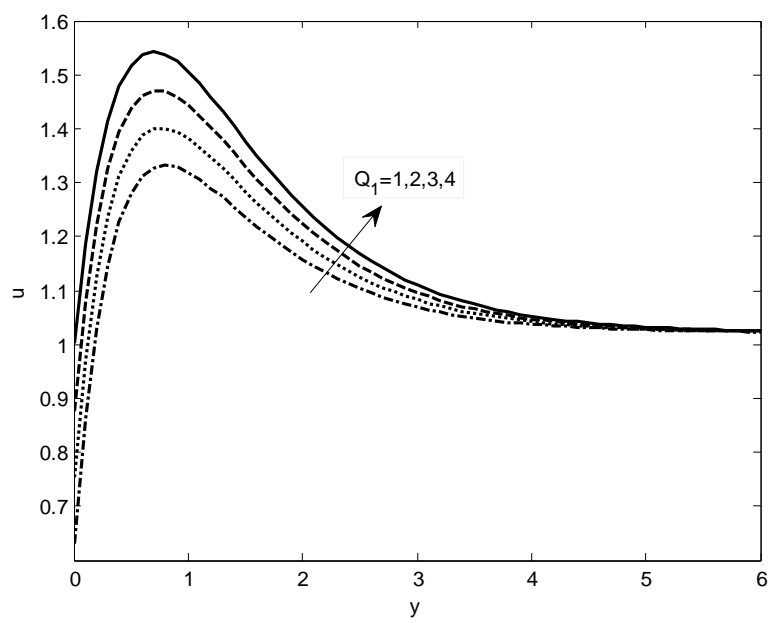

Fig.1 Velocity profiles for different values of $Q_{1}$ with $G r=4, G m=2, D u=0.3, \emptyset_{1}=0.3$,

$$
F=2, \gamma=1, M=2, \emptyset=1, \alpha=\frac{\pi}{6}, \psi=\frac{\pi}{4}
$$

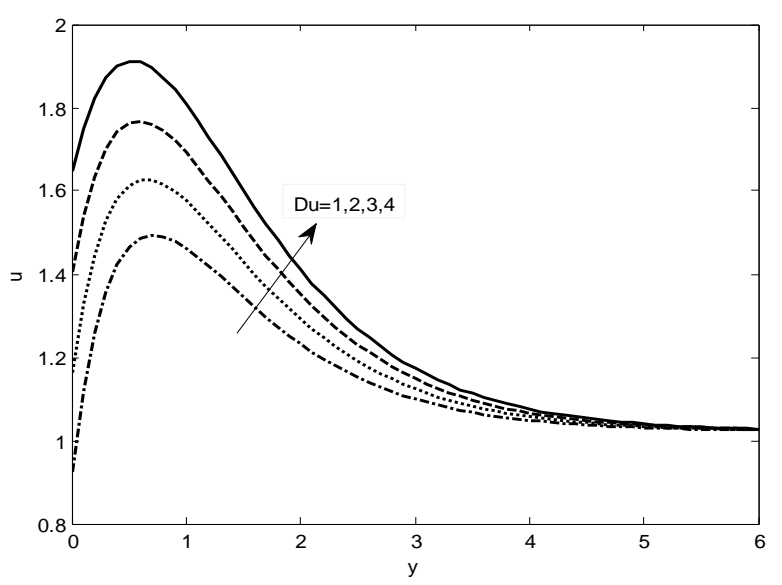

Fig.2 Velocity profiles for different values of $D u$ with $G r=4, G m=2, Q_{1}=2, \emptyset_{1}=0.3$,

$$
F=2, \gamma=1, M=2, \emptyset=1, \alpha=\frac{\pi}{6}, \psi=\frac{\pi}{4} .
$$




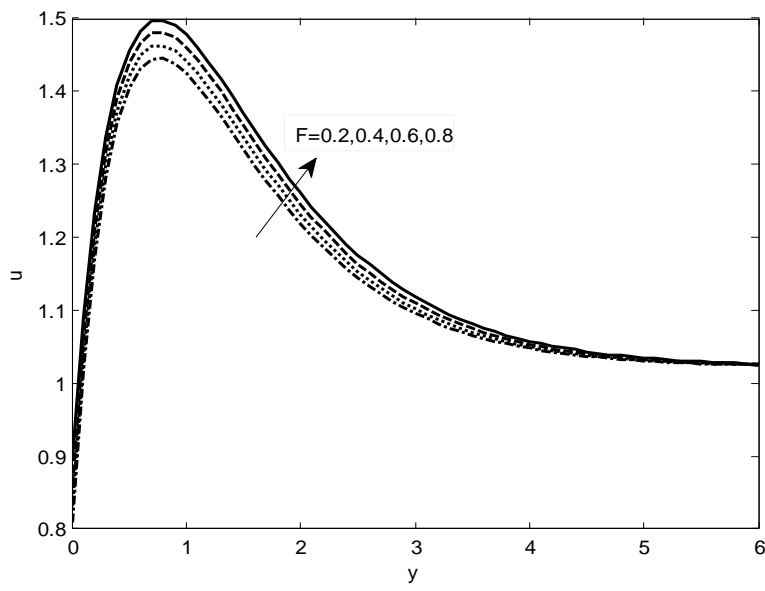

Fig.3.Velocity profiles for different values of $F$ with $G r=4, G m=2, Q_{1}=1, \emptyset_{1}=0.3, \psi=\frac{\pi}{4}$

$$
D u=0.5, \gamma=1, M=2, \varnothing=1, \alpha=\frac{\pi}{6}
$$

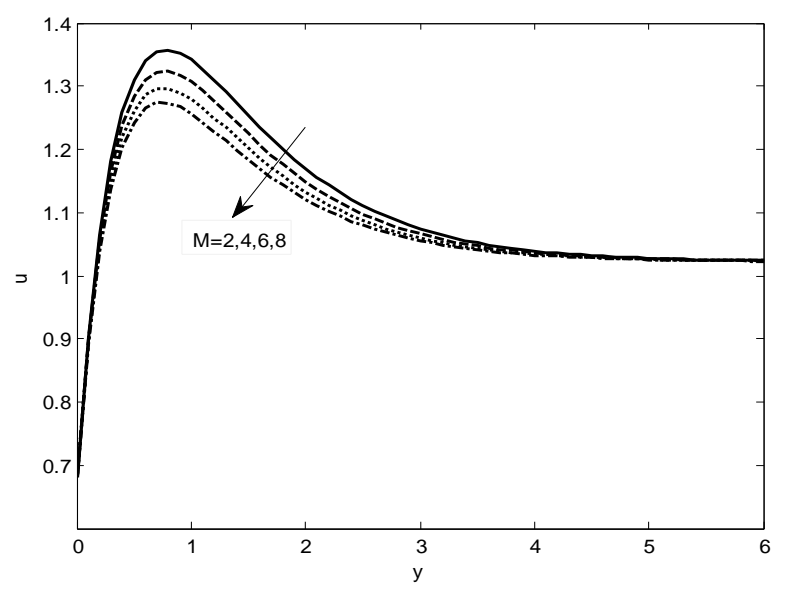

Fig.4 Velocity profiles for different values of $M$ with $G r=4, G m=2, Q_{1}=2, \emptyset_{1}=0.3, F=2, \gamma=1, D u=$ $0.5, \varnothing=1, \alpha=\frac{\pi}{6}, \psi=\frac{\pi}{4}$.

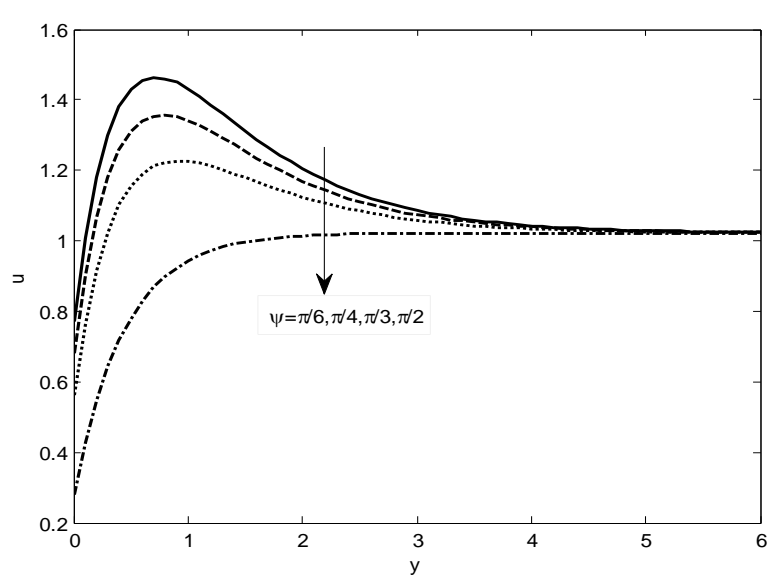

Fig.5. Velocity profiles for different values of $\psi$ with $Q_{1}=1, D u=0.5, \alpha=\frac{\pi}{6}$. $\emptyset_{1}=0.3$,

$$
F=2, \gamma=1, M=2, \emptyset=1 \text {, }
$$




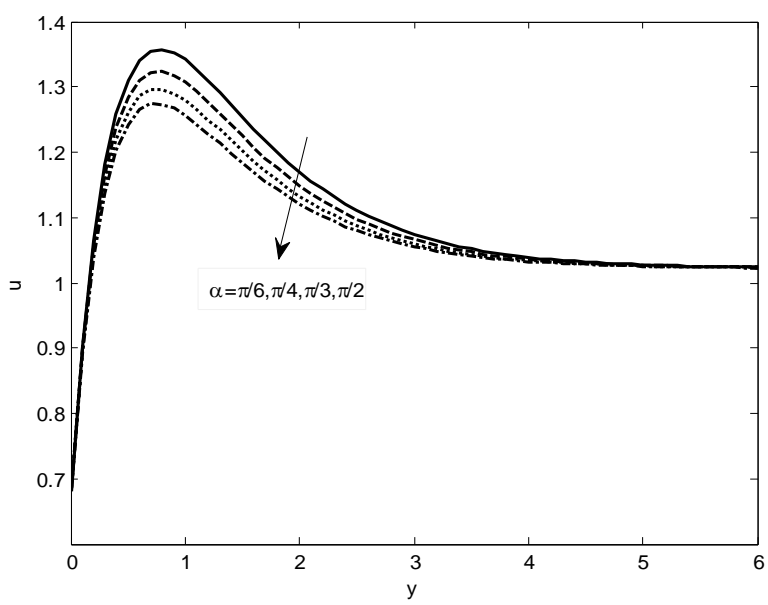

Fig.6.Velocity profiles for different values of $\alpha$ with $Q_{1}=1, \emptyset_{1}=0.3, F=2, \gamma=1, M=2, \emptyset=1, \psi=\frac{\pi}{4}$.

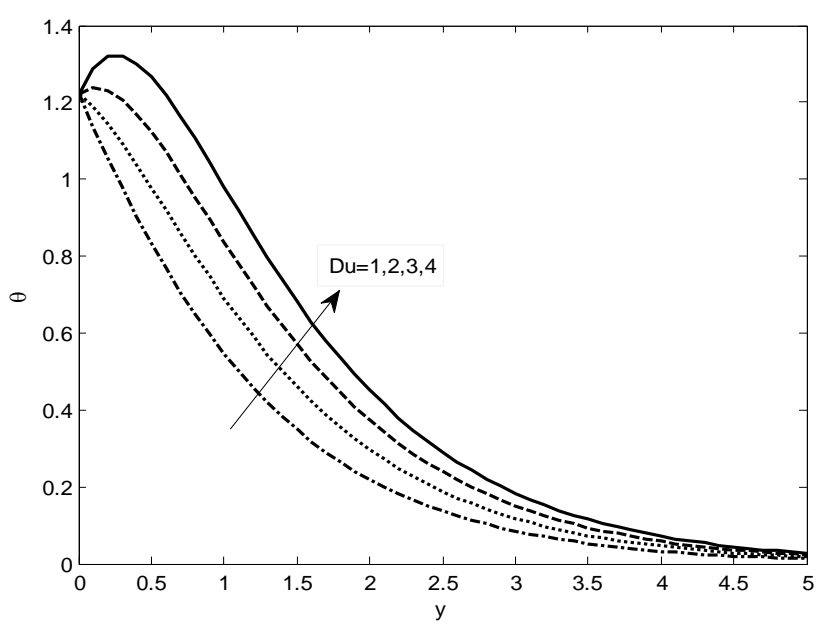

Fig.7 Temperature profiles in $D u$ with $\gamma=0.5, Q_{1}=2$.

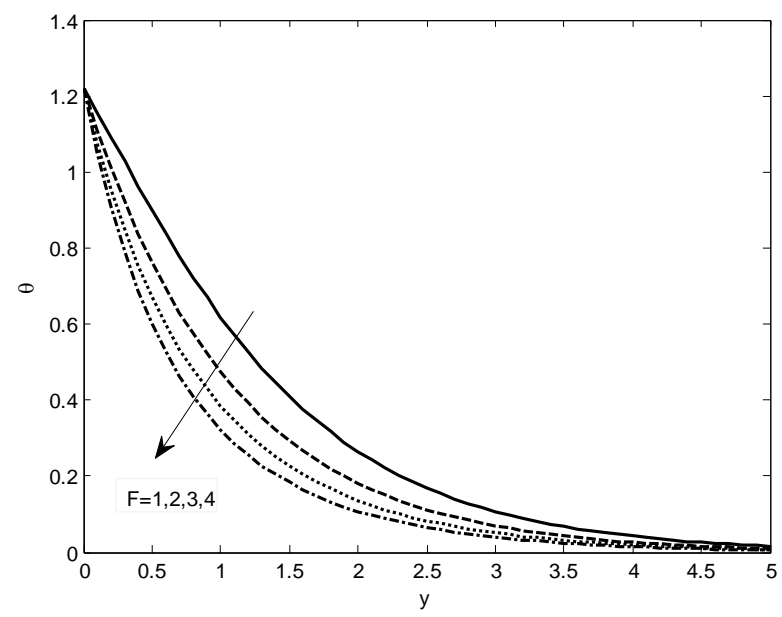


Fig.8 Temperature profiles in F with $G r=4, G m=2, \gamma=0.5, Q_{1}=2, D u=0.5$

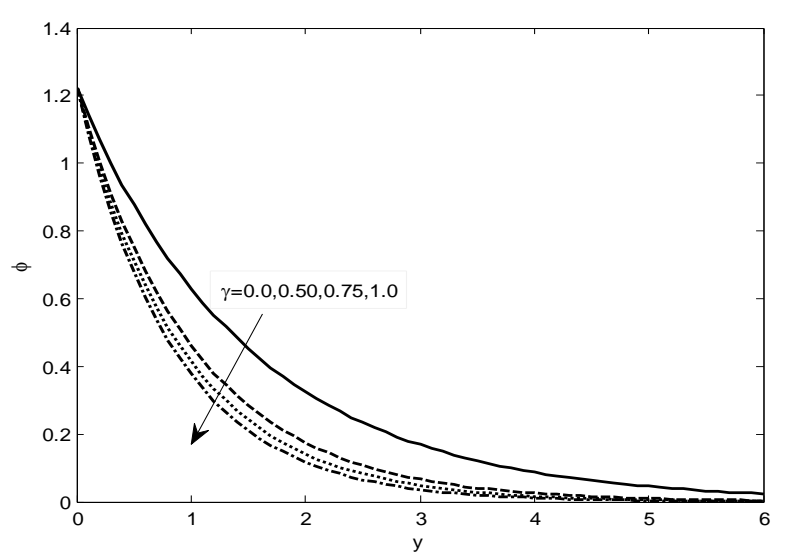

Fig.9 Concentration profiles in Sc with $G r=4, G m=2, \gamma=0.5, Q_{1}=2$

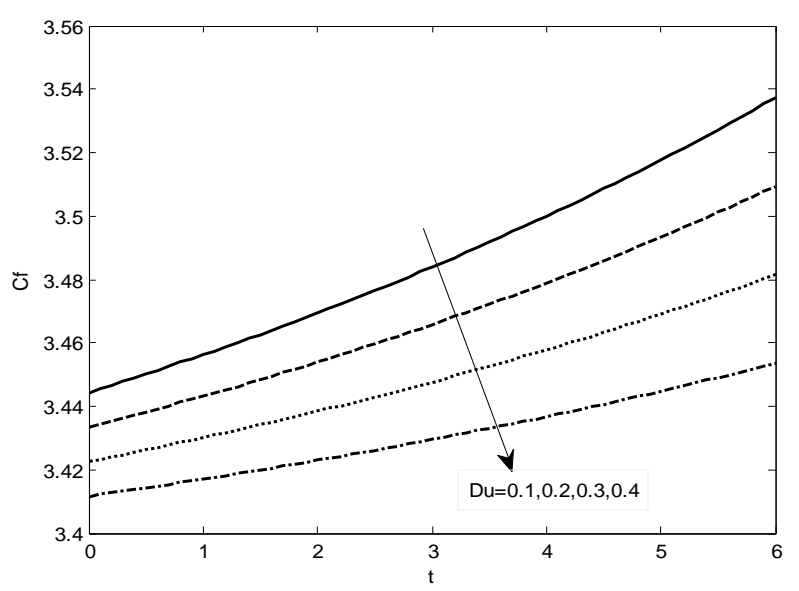

Fig.10 Skin-friction profiles for different values of Du with $Q_{1}=2, \emptyset_{1}=0.3, F=2, \gamma=1, M=2, \emptyset=1$, $\alpha=\frac{\pi}{6}, \psi=\frac{\pi}{4}$.

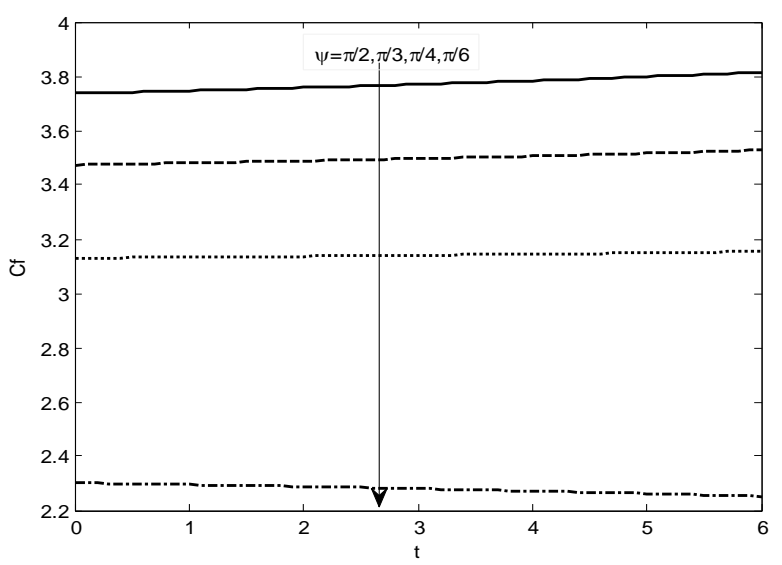

Fig.11 Skin-friction profiles for different values of $\psi$ with $G r=4, G m=2, Q_{1}=2$, 


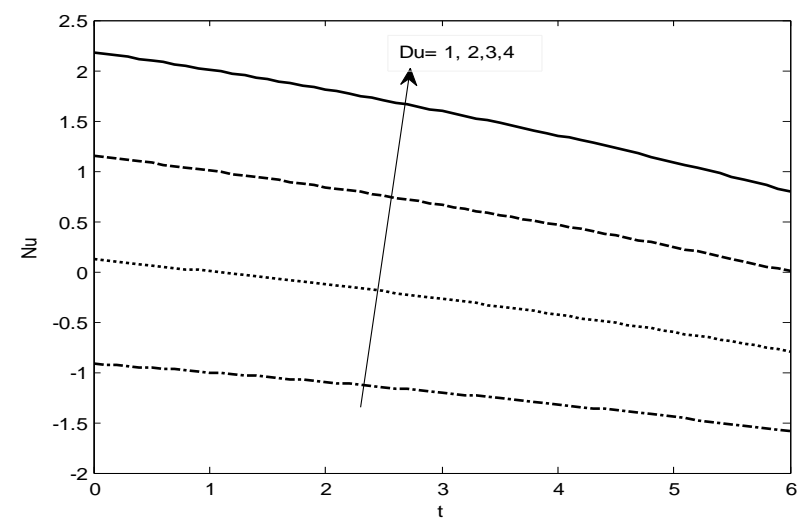

Fig.12 Local Nusselt number for various values of $D u$ against time $\mathrm{t}$ with $Q_{1}=2, \gamma=5, \varnothing=1$.

Fig.1 represents the graph of velocity profile for different values of radiation absorption parameter $\left(Q_{1}\right)$. It is clear from the figure that an increase in the absorption radiation parameter $\left(Q_{1}\right)$ results an increasing in the velocity profiles within the boundary layer as well as an increasing in the momentum and thermal thickness this is because the large $Q_{1}$ values correspond to an increased dominate of conduction over absorption radiation thereby increasing buoyancy force and thickness of the momentum boundary layer. The influence of Dufour number on the velocity distribution across the boundary layer is shown in Fig.2. The results indicate that with increase in the parameter Du, the velocity of the fluid increases. Thus the effect of Du causes to increase the boundary layer thickness and hence the maximum peak value hydrodynamic attains near the plate. The effect of radiation parameter on the horizontal velocity in the momentum boundary layer is shown in Fig.3. We note from this figure that there is in the horizontal velocity profiles with increase in the radiation parameter $(F)$. The increase of the radiation parameter $(F)$ leads to enhance the heat transfer rate in the presence of thermal and solutal buoyancy force. The effects of the magnetic field parameter $(M)$ on the velocity is presented in Fig.4. The increase in the value of $M$ causes the reduction in velocity profiles across the boundary layer. Physically, the higher value of $M$ implies the higher Lorentz force which has tendency to slow down the motion of the conducting fluid in the boundary layer. Fig.5. shows the effect of aligned angle $\psi$ on velocity profiles. From this figure it is clear that an increase in aligned angle $(\psi)$ decreases the velocity profiles. The reason behind this is increase in aligned angle causes to strength in the magneticfield. Due to enhanced magneticfield, it generates opposite force to the flow, is called Lorentz force. This force reduces the thickness of the momentum boundary layer. Fig.6 indicates that the effect of inclination angle $\alpha$ on the fluid velocity. It is observed the fluid velocity is decreases with an increasing value of $\alpha$. The decreasing rate of inclination causes to increase the fluid velocity. It can be concluded that higher angle of the inclined surface denotes greater gravity force acting on the surface. Fig.7 presents the effect of diffusion-thermo parameter $(D u)$ on temperature distribution is increases.

Fig.8 shows the temperature profiles for different values of the thermal radiation parameter $(F)$. For large $F$, it is observed that temperature decreases more rapidly with the increase of radiation parameter $F$. Therefore using radiation we can control the flow characteristic and temperature distribution. For different values of destructive chemical reaction parameter $\gamma(>0)$, the concentration profiles are plotted in Fig.9. An increase in chemical reaction parameter will suppress the concentration of the fluid. Higher values of $\gamma$ amount to a fall in the chemical molecular diffusivity, i.e., less diffusion. Therefore, they are obtained by species transfer. An increase in $\gamma$ will suppress species concentration. The concentration distribution decreases at all points of the flow field with the increase in the reaction parameter.

Figs. 10and11 shows the Skin-friction against time $t$ for various values of Dufour number (Du), and aligned angle $(\psi)$. It is noticed that the friction at the wall deceases with an increasing values of Dufour number, inclination angle and aligned angle. Fig. 12 reveals the effect of Nusselt number against time $t$ for various values of fluid flow parameter $D u$. It can be seen that the rate of heat transfer coefficient increases with increasing values of $D u$.

\section{V.Comparison of results}


In order to examine the accuracy of the results of the present study, it is considered that the analytical solutions obtained by Kim [15] and Pal and Talukdar [16] who computed the numerical results for Skin friction coefficient and Nusselt number. These computed and compared results are presented in table-1. From this table it is interesting to observe that the present results in the absence of Diffusion-thermo effect and Porous medium when $\alpha=0$ are in good agreement with the corresponding results obtained from Pal and Talukdar [16]. It is also observed that the present results are in good agreement with those of $\operatorname{Kim}[15]$ when $G=2, u_{p}=0, D u=$ $0, G m=0, \alpha=0$, and $\psi=\frac{\pi}{2}$.

Table.1: Comparison of present results with those of Kim [15] and Dulal Pal [16] with different values of

$$
C_{f x}, N u_{x} / R e_{x}
$$

\begin{tabular}{llllll}
\hline Kim[15] & & Pal and Talukdar [16] \\
& & &
\end{tabular}

\section{References}

[1]. R.J. Gribben, "The Magneto hydrodynamic boundary layer in the presence of pressure gradient". Proc.Royel.Soclondon.1965.Vol. A287, pp. 123-141.

[2]. Ali F, Khan I, Samiulhaq, S.Shafie. "Conjugate Effects of Heat and Mass Transfer on MHD Free Convection Flow over an Inclined Plate Embedded in a Porous Medium". PLoS ONE. 2013 Vol.8, pp.1-11.

[3]. G. Palani. "Convection effects on flow past an inclined plate with variable surface temperatures in water at $4{ }^{\circ} \mathrm{C}$ ". Ann Faculty Eng Hunedora. 2008, Vol.1. pp.75-82

[4]. R.N. Barik, G.C. Dash, P.K. Rath. "Thermal Radiation effect on an unsteady MHD flow past inclined porous heated plate in the presence of chemical reaction and viscous-dissipation".ApplMath Comput.2014. Vol.226, pp.423-434.

[5]. C.H. Chen, "Heat and mass transfer in MHD flow by natural convection from a permeable inclined surface with variable wall temperature and concentration". Acta Mech.2004. Vol.172, pp.219-235.

[6]. Sudersan Reddy, G.V. Ramana Reddy, K. Jayarami Reddy, "Radiation and chemical reaction effects on MHD heat and mass transfer flow inclined porous heated plate". Asian J. Curr. Eng. Math.2012.Vol. 1. pp.115-119.

[7]. Ganesan, G. Palani, "Finite difference analysis of unsteady natural convection MHD flow past an inclined plate with variable surface heat and mass flux". Int J Heat Mass Transfer.2004.Vol. 47, pp.4449-4457.

[8]. V.Prabhakara Reddy, R.V.M.S.S.Kiran Kumar, G. Viswanatha Reddy, P. Durga Prasad, and S.V.K. Varma. "Free Convection Heat and Mass Transfer Flow of Chemically Reactive and Radiation Absorption Fluid in an Aligned Magnetic Filed". Procedia Engineering. 2015.Vol. 127, pp.575-582.

[9]. H.S. Takhar, A.J. Chamkha, and G. Nath. "Unsteady Flow and Heat Transfer on a Semi-infinite Flat Plate with an Aligned Magnetic Field". International Journal of Engineering Science, 1999.Vol.37, pp.1723-173.

[10]. D.A.Nield and A.Bejan, "Convection in Porous Media". $2^{\text {nd }}$ Edition, Springer Verlag, Berlin, 1999.

[11]. M.G.Reddy, N.B. Reddy. "Mass transfer and heat generation effects on MHD free convection flow past an inclined vertical surface in a porous medium". 2011,Vol.4,.pp.7-11.

[12]. E.R.G.Eckert, R.M.Drake, "Analysis of Heat and Mass Transfer". M.C.Graw-Hill, New York, 1972.

[13]. M.Anghel, H.S Takhar, I.Pop, "Dufour and Soret effects on free convection boundary layer over a vertical surface embedded in a porous medium". Studia Universitatis Babes-Bolyai, Cluj-Napoca, 2000, Vol.4,pp.11-21.

[14]. Z. Dursunkaya, W.M.Worek. "Diffusion-thermo and thermal-diffusion effects in transient and steady natural convection from vertical surface". Int. J. Heat Mass Transf. 1992.Vol. 35, pp.2060-2065.

[15]. Y.J. Kim, "Unsteady MHD convective heat transfer past a semi-infinite vertical moving plate with variable suction". Int J Eng. Sci. 2000, Vol.38, pp.833-45.

[16]. Dulal Pal, Babulal Talukdar. "Perturbation analysis of unsteady magnetohydrodynamic convective heat and mass transfer in a boundary layer slip flow past a vertical permeable plate with thermal radiation and chemical reaction". Commun Nonlinear Sci Numer Simulat.2010. Vol 15, pp.1813-1830.

[17]. P. Durga Prasad, R.V.M.S.S Kiran Kumar, B.Mamatha, S.V.K. Varma. "Diffusion-thermo and porous medium effects on MHD free convection heat and mass transfer flow over an inclined surface", Global Journal of Pure and Applied Mathematics. 2016. Vol 12. pp. $142-150$. 